\title{
ORIGINAL
}

\section{PÉRDIDAS DE PRODUCCIÓN LABORAL OCASIONADAS POR LOS FALLECIMIENTOS PREMATUROS EN ESPAÑA EN EL AÑO 2005 (*)}

\author{
Juan Oliva $(1,2,3)$ \\ (1) Universidad de Castilla la Mancha-Dpto. Análisis Económico y Finanzas. \\ (2) Fundación de Estudios de Economía Aplicada (FEDEA). \\ (3) CIBER de Epidemiología y Salud Pública (CIBERESP).
}

\section{RESUMEN}

Fundamentos: Conjuntamente con las muertes, las pérdidas en la calidad de vida y el dolor de pacientes y familiares que ocasionan las enfermedades y los accidentes, existen otros indicadores que ayudan a una mayor compresión su impacto social. El objetivo del presente trabajo es estimar una parte del impacto no sanitario que enfermedades y accidentes ocasionan en la economía.

Métodos: En el análisis se estiman las pérdidas de producción que provocaron las muertes prematuras en España acaecidas durante el año 2005. Empleando las estadísticas disponibles, Registro de Defunciones según la causa de muerte, Encuesta de Población Activa y Encuesta de Estructura Salarial, se desarrolla un modelo de simulación basado en el enfoque del capital humano.

Resultados: se estimó una pérdida de producción superior a los 9.100 millones de euros a consecuencia de las muertes prematuras acaecidas en España durante el año 2005. Esta cifra fue equivalente al 1,01\% del Producto Interior Bruto de España en el año 2005, o a un 18,25\% del gasto sanitario público del mismo año.

Conclusiones: La mortalidad prematura genera un importante coste laboral

Palabras clave: Economía de la salud. Análisis de costes. Costes de las enfermedades. Mortalidad prematura. Causa de muerte.

\section{ABSTRACT \\ Loss of Labour Productivity Caused by Premature Mortality in Spain in 2005}

Background: Jointly with deaths, loss of quality of life and pain of patients and families, there are complementary indicators that help to reveal the social impact caused by illnesses and accidents. In the analysis, loss of labour productivity due to premature mortality in 2005 is estimated. The aim of this work is to estimate a part of the non health care costs caused by illnesses and accidents in the economy.

Methods: A simulation model based in the human capital approach is developed using several available Spanish data bases (Death Registry According to Cause of Death, Structural Wage Survey, Labour Force Survey).

Results: A loss of production up to 9,100 millions of euros due to premature mortality is estimated in Spain for the base year. This figure is equivalent to a $1.01 \%$ of the Spanish Gross Domestic Product, or to an $18.25 \%$ of the Public Health Care Expenditure of 2005 .

Conclusions: Premature mortality caused a very high labour cost.

Key words: Health economics. Costs and Cost Analysis. Cost of illness. Mortality. Cause of Death.
Correspondencia:

Juan Oliva

Universidad de Castilla la Mancha

Facultad de Ciencias Jurídicas y Sociales de Toledo

Análisis Económico y Finanzas

Cobertizo de San Pedro Mártir s/n. 45071. Toledo

Correo electrónico: juan.olivamoreno@uclm.es
(*) Este trabajo ha recibido una ayuda del Instituto de Estudios Fiscales para su elaboración. Asimismo, también se beneficia del apoyo del proyecto "Individual decisions in dynamic contexts, welfare effects and evaluation of public policies- Dinámica en las decisiones individuales, efectos sobre el bienestar y evaluación de políticas públicas" financiado por el Ministerio de Ciencia e Innovación (ECO2008-06395-C05-03/ECON). 


\section{INTRODUCCIÓN}

Conjuntamente con las muertes, las pérdidas en la calidad de vida y el dolor de pacientes y familiares que ocasionan las enfermedades y los problemas de salud, es posible identificar otros indicadores que ayuden a una mayor compresión de su impacto social. Por ejemplo, se pueden identificar, medir y valorar los recursos empleados en la prevención y tratamiento del problema de salud en cuestión. Así, se denominan costes directos sanitarios a aquellas inversiones en recursos en atención primaria, atención especializada, hospitalizaciones, medicamentos y programas de carácter preventivo que tratan de reducir en lo posible el impacto en la salud de las personas que sufren o podrían sufrir una enfermedad. Sin embargo, existen otros costes adicionales que merecen atención desde la perspectiva social.

Un aspecto a destacar es que el término gasto sanitario puede ser intercambiado en numerosas situaciones por el de inversión sanitaria. El resultado que obtendríamos a cambio de los recursos sanitarios asignados (o invertidos) sería una ganancia en términos de cantidad o de calidad de vida (o de ambas) y evitar otra serie de costes relacionados con las enfermedades. Así, el tener una buena salud tiene un doble componente: consumo e inversión ${ }^{1,2}$. Además de la valoración intrínseca que hacemos los individuos sobre este aspecto de consumo: "gozar de buena salud", una mejora en el estado de salud aumenta la productividad del trabajo e incrementa las oportunidades de obtener mejores salarios. En este sentido, la salud puede entenderse como una dimensión del capital humano de las sociedades, donde inversiones presentes, dirigidas a mejorar la salud de las poblaciones, se materializarían en un futuro en forma de crecimiento económico de las mismas ${ }^{3}$.

Por esta razón, una asignación de recursos sanitarios tendrá como primer y claro objetivo mejorar la esperanza y la calidad de la vida de los ciudadanos que conforman la sociedad. Sin embargo, complementariamente no se deberían dejar de considerar otros componentes de la pérdida de bienestar social que ocasionan las enfermedades. Para ello, se debe tomar en cuenta la existencia de costes no sanitarios de la enfermedad puesto que pese a no tener un reflejo sobre partidas presupuestarias ni incluirse estas pérdidas, al menos de manera directa, sobre las cifras de la Contabilidad Nacional sí estarán reflejando una importante pérdida de bienestar social.

Recientemente la Comisión Europea ha reconocido la importancia de la salud en el desarrollo económico próximo de la Unión Europea (UE), de tal modo que uno de los indicadores clave de crecimiento económico propuesto en la Estrategia de Lisboa para el Crecimiento y el Empleo del año $2005^{4}$ fueron los Años de Vida Saludables (Healthy Life Years). Más recientemente, Together for Health, la estrategia de política sanitaria para la UE propuesta por la Comisión Europea para el periodo 20082013, subraya este hecho ${ }^{5}$.

El objetivo de este trabajo es aportar información objetiva y comparable sobre aspectos relacionados con el coste de las enfermedades y problemas de salud. La idea fundamental es que la información aportada, junto con otros datos de carácter económico, presupuestario y sanitario, se integre en un cuerpo de información de utilidad para el planificador público. Más concretamente, en el presente papel se estimará el coste social que representan las pérdidas de productividad laboral ocasionadas por los fallecimientos prematuros acaecidos en España durante el año 2005.

En cuanto a la utilidad del presente trabajo, generalmente las críticas hacia los estudios de costes de la enfermedad se basan en que lo que importa no es el coste total de la enfermedad sino lo que se puede 
hacer para evitarla o mitigarla y los recursos necesarios para ello y que, por ello, la estimación de los costes totales de una enfermedad son útiles como medida de los beneficios de programas de prevención y tratamiento sólo si las intervenciones son capaces de prevenir o erradicar la enfermedad (análisis marginal).

$\mathrm{Si}$ bien es cierto que los recursos no deberían asignarse en función del impacto de una determinada enfermedad, sino allí donde mayores beneficios en términos de salud produzca una intervención, los estudios de costes permiten en muchos casos presentar la verdadera dimensión de un problema de salud, aportar una información valiosa para la sociedad y los decisores en relación a la importancia relativa y absoluta de dicha enfermedad y, por tanto, ayudar a la correcta asignación de prioridades partiendo de los recursos sanitarios y sociales disponibles. Así, la utilidad de un estudio de costes consiste en señalar el impacto económico de una o varias enfermedades y problemas de salud, al contabilizar una parte importante de la carga social que conlleva la mortalidad y la morbilidad de las mismas.

\section{MATERIAL Y MÉTODOS}

El concepto de productividad laboral se realiza a través de una aproximación. Esto es, dada la dificultad de medición del concepto, y basándonos en las teorías económicas del capital humano ${ }^{1,2,6-10}$, se considera que una variable razonable de la productividad de un individuo es el salario bruto (o ganancia salarial) que éste obtiene.

El salario bruto medio es obtenido de la Encuesta de Estructura Salarial (EES) del año 2005 del Instituto Nacional de Estadística (INE). Este concepto se refiere a las remuneraciones en metálico y en especie, pagadas a los trabajadores por el tiempo trabajado o por el trabajo realizado, junto a la remuneración por períodos de tiempo no trabajados, como vacaciones y días festivos. Se computan los devengos brutos, es decir, antes de haber practicado las deducciones de las aportaciones a la Seguridad Social por cuenta del trabajador o las retenciones a cuenta del Impuesto sobre la Renta de las Personas Físicas.

Los datos sobre ocupación se obtuvieron de la Encuesta de Población Activa (EPA) del INE realizada durante al año 2005. La población ocupada queda definida como aquellas personas de 16 o más años que durante la semana de referencia han estado trabajando durante al menos una hora, a cambio de una retribución (salario, jornal, beneficio empresarial, etcétera) en dinero o especie. También son ocupados quienes teniendo trabajo han estado temporalmente ausentes del mismo por enfermedad, vacaciones, etcétera. La tasa de ocupación o tasa de empleo se define como el porcentaje de la población ocupada en relación con la población en edad de trabajar.

Los datos necesarios para el cálculo de las muertes y los Años Potenciales de Vida Perdidos se encuentran en el Registro de Defunciones según la Causa de Muerte correspondiente al año 2005, publicados por el Instituto Nacional de Estadística. Dicha fuente proporciona información anual sobre los fallecimientos acaecidos dentro del territorio nacional atendiendo a la causa básica que los determinó y distinguiendo por Comunidad Autónoma de residencia. Para ello se emplea la Clasificación Internacional de Enfermedades de la Organización Mundial de la Salud, utilizándose a partir de 1999 la $10^{a}$ revisión de dicha clasificación.

Una vez que se conoce la edad de fallecimiento de cada individuo y los salarios esperados, se realiza el cálculo del flujo presente y futuro de la productividad laboral perdida por una muerte prematura ocasionada por cualquiera de las causas consi- 
deradas. Con este fin, para cada muerte producida en un grupo de edad y género determinado, se irá aplicando la tasa de ocupación y ganancia salarial esperados a cada periodo posterior hasta un límite prefijado (en nuestro caso, los 65 años). A los valores futuros obtenidos se les aplicó una tasa anual de descuento del tres por ciento y una tasa anual de crecimiento de la productividad laboral del uno por ciento. Este es el caso base, al cual se aplica un análisis de sensibilidad. Para ello se consideran dos tasas de descuento alternativas, cero por ciento y seis por ciento, y dos nuevas tasas de crecimiento de la productividad laboral, cero por ciento y dos por ciento.

Junto con el cálculo de la pérdida laboral, se aporta información sobre otros dos indicadores medidos en unidades físicas: los Años Potenciales de Vida Perdidos (APVP) y los Años Potenciales de Vida Laboral Perdidos (APVLP). Estos indicadores aportan información complementaria al cálculo de la pérdida laboral, estando más cerca del concepto de la carga de la enfermedad que del impacto económico de la pérdida laboral. En todo caso los tres elementos, pérdidas laborales, APVP y APVLP, son indicadores (imperfectos) del coste social de los fallecimientos prematuros.

Para realizar el cálculo de los APVP se sigue el mismo proceso que el consignado en las notas metodológicas recogidas por el Instituto Nacional de Estadística (INE). Este indicador representa una medida de mortalidad que teóricamente se podría evitar. Para ello tiene en cuenta los años que una persona deja de vivir si fallece a una edad que no es la habitual de defunción fijada teóricamente para ese colectivo. El cálculo de este indicador se realiza para el intervalo de edad comprendido entre 1 y 69 años, lo que supone prescindir, por un lado, de las muertes ocurridas en las edades más avanzadas $\mathrm{y}$, por otro, de la mortalidad infantil debido a que las causas de muerte de los fallecidos menores de 1 año son, en general, muy específicas, requiriendo un estudio aparte.

Los APVLP se calculan a partir del número de fallecimientos en edad laboral o en edad previa al momento de acceso al mercado de trabajo (menores de 16 años). Se consideró como edad límite de permanencia en el mercado de trabajo, la edad legal de jubilación, es decir, los 65 años. Ello implica que los APVLP son igual a 49 para cada fallecimiento producido a la edad de 16 años o más temprana y que los APVLP son igual a cero para cada fallecimiento producido a una edad igual o superior a los 65 años. Por tanto, a diferencia de los APVP, este indicador recoge los fallecimientos de los niños menores a un año, pero excluye los fallecimientos producidos a los 65 o más años.

\section{RESULTADOS}

El número de fallecimientos totales en España acaecidos durante el año 2005 ascendió a 387.355 personas. De estas muertes, un $52 \%$ corresponden a varones y un $48 \%$ a mujeres. Los APVP se estiman en 1,25 millones, observándose importantes diferencias por sexo. Así, los varones asumen el $71 \%$ de los años potenciales de vida perdidos, mientras que las mujeres pierden el $29 \%$ restante. Casi las $3 / 4$ partes de las diferencias en APVP entre varones y mujeres se pueden explicar por el mayor número de muertes prematuras que sufren los varones por causas externas, tumores y enfermedades circulatorias, por orden de importancia (tabla 1).

Se produjo un total de 65.441 muertes en edad laboral o previa, 46.309 correspondieron a varones (el 70,8\%) y 19.132 a mujeres $(29,2 \%)$. En total se perdieron algo más de un millón de Años Potenciales de Vida Laboral (APVLP), correspondiendo el 70\% a varones y el $30 \%$ restante a mujeres. 
Tabla 1

Número de fallecimientos (España, año 2005)

\begin{tabular}{|l|c|c|c|c|c|c|}
\hline & De 0 a 24 años & De 25 a 44 años & De 45 a 64 años & De 65 a 74 años & 75 años y más & TOTAL \\
\hline $\mathrm{N}^{\circ}$ fallecimientos & 4.999 & 13.487 & 46.955 & 64.267 & 257.647 & 387.355 \\
\hline Varones & 3.311 & 9.644 & 33.354 & 42.516 & 112.944 & 201.769 \\
\hline Mujeres & 1.688 & 3.843 & 13.601 & 21.751 & 144.703 & 185.586 \\
\hline
\end{tabular}

Fuente: Elaboración propia a partir del Registro de Defunciones por Causa de Muerte.

Tabla 2

Número de fallecimientos de menores de 65 años y Años Potenciales de Vida Laboral Perdidos en España (año 2005)

\begin{tabular}{|l|c|c|c|}
\hline & Varones & Mujeres & Total \\
\hline $\mathrm{N}^{\circ}$ fallecimientos de menores de 65 años & 46.309 & 19.132 & 65.441 \\
\hline Años Potenciales de Vida Laboral Perdidos (APVLP) & 720.968 & 308.585 & 1.029 .553 \\
\hline
\end{tabular}

Fuente: Elaboración propia a partir del Registro de Defunciones por Causa de Muerte.

Por lo que respecta a los fallecimientos, los dos grupos de enfermedades que mayores muertes provocan son las enfermedades del sistema circulatorio y los tumores, siendo responsables del $33 \%$ y del $26 \%$ de los decesos, respectivamente. A continuación se encontrarían las enfermedades respiratorias $(12 \%)$ y varios grupos de enfermedades que se sitúan en torno al 3-5\% (enfermedades mentales, enfermedades del sistema nervioso, enfermedades del sistema digestivo y causas externas de mortalidad).

En cuanto a los fallecimientos de personas menores de 65 años, los tumores destacan sobre el resto, siendo responsables del $40 \%$ de los fallecimientos, seguidos por las enfermedades del sistema circulatorio $(18 \%)$ y por las causas externas de mortalidad (15\%), las enfermedades del sistema digestivo (6\%), las enfermedades respiratorias $(5 \%)$ y las enfermedades infecciosas y parasitarias (debido al peso del VIH/SIDA) $(4 \%)$.

Si nos centramos en los APVP, un 34\% del total corresponden a tumores, pasando a ser el segundo grupo de mayor peso pasa a ser las causas externas de mortalidad, con un $24 \%$ de los APVP, seguidas de las enfermedades del sistema circulatorio, con un
$16 \%$. A más distancia quedan las enfermedades del sistema digestivo, las enfermedades infecciosas y parasitarias y las enfermedades respiratorias.

Se estima que un $29 \%$ de los APVLP corresponden a tumores, seguido a corta distancia, de las causas externas de mortalidad, con un $25 \%$. En tercer lugar aparecen las enfermedades del sistema circulatorio (13\%) y, a más distancia, las enfermedades infecciosas y parasitarias $(5,0 \%)$, las enfermedades del sistema digestivo $(4,9 \%)$ y las enfermedades respiratorias $(4,0 \%)$. Los fallecimientos por afecciones originadas en el periodo perinatal implican una fuerte pérdida de APVLP, debido a la corta edad de los fallecidos, suponiendo un $4,45 \%$ de todos los APVLP (tabla 3).

Las pérdidas de productividad laboral ocasionadas por muertes prematuras en España en el año 2005 se han estimado en 9.136 millones de euros. El caso base escogido es aquel que considera una tasa de descuento anual del 3\% y asume una tasa de crecimiento anual de la productividad laboral del $1 \%$. Hay dos grupos que destacan sobre el resto y que conjuntamente suponen más del $50 \%$ de las pérdidas de productividad laboral: causas 
Tabla 3

Principales indicadores derivados de los fallecimientos. Valores absolutos y distribución según causa del fallecimiento

\begin{tabular}{|c|c|c|c|c|c|c|c|c|}
\hline \multirow[b]{2}{*}{ 001-102 I-XX. Todas las causas } & \multicolumn{2}{|c|}{ Fallecimientos } & \multicolumn{2}{|c|}{$\begin{array}{l}\text { Fallecimientos en } \\
\text { menores de } 65 \text { años }\end{array}$} & \multicolumn{2}{|c|}{ APVP } & \multicolumn{2}{|c|}{ APVLP } \\
\hline & 387.355 & $100,00 \%$ & 65.441 & $100,00 \%$ & 1.253 .529 & $100,00 \%$ & 1.029 .553 & $100,00 \%$ \\
\hline 001-008 I. Enfermedades infecciosas y parasitarias (1) & 7.493 & $1,93 \%$ & 2.536 & $3,88 \%$ & 61.307 & $4,89 \%$ & 51.528 & $5,00 \%$ \\
\hline 009-041 II. Tumores & 100.206 & $25,87 \%$ & 26.359 & $40,28 \%$ & 429.283 & $34,25 \%$ & 302.089 & $29,34 \%$ \\
\hline $\begin{array}{l}\text { 042-043 III. Enf. de sangre y de órg. hematopoyéticos y } \\
\text { ciertos trast. que afectan mecan. inmunidad }\end{array}$ & 1.316 & $0,34 \%$ & 187 & $0,29 \%$ & 4.687 & $0,37 \%$ & 4.122 & $0,40 \%$ \\
\hline $\begin{array}{l}\text { 044-045 IV. Enfermedades endocrinas, nutricionales y } \\
\text { metabólicas }\end{array}$ & 12.421 & $3,21 \%$ & 1.159 & $1,77 \%$ & 19.719 & $1,57 \%$ & 15.555 & $1,51 \%$ \\
\hline 046-049 V. Trastornos mentales y del comportamiento & 12.539 & $3,24 \%$ & 478 & $0,73 \%$ & 10.571 & $0,84 \%$ & 8.295 & $0,81 \%$ \\
\hline $\begin{array}{l}\text { 050-052 VI-VIII. Enfermedades del sistema nervioso y de } \\
\text { los órganos de los sentidos }\end{array}$ & 15.697 & $4,05 \%$ & 1.675 & $2,56 \%$ & 39.740 & $3,17 \%$ & 33.919 & $3,29 \%$ \\
\hline 053-061 IX. Enfermedades del sistema circulatorio & 126.907 & $32,76 \%$ & 11.742 & $17,94 \%$ & 194.788 & $15,54 \%$ & 136.249 & $13,23 \%$ \\
\hline 062-067 X. Enfermedades del sistema respiratorio & 47.089 & $12,16 \%$ & 3.087 & $4,72 \%$ & 56.574 & $4,51 \%$ & 41.628 & $4,04 \%$ \\
\hline 068-072 XI. Enfermedades del sistema digestivo & 19.624 & $5,07 \%$ & 4.015 & $6,14 \%$ & 69.359 & $5,53 \%$ & 50.780 & $4,93 \%$ \\
\hline 073 XII. Enfermedades de la piel y del tejido subcutáneo & 1.107 & $0,29 \%$ & 50 & $0,08 \%$ & 896 & $0,07 \%$ & 640 & $0,06 \%$ \\
\hline $\begin{array}{l}\text { 074-076 XIII. Enfermedades del sistema osteomuscular y } \\
\text { del tejido conjuntivo }\end{array}$ & 3.596 & $0,93 \%$ & 226 & $0,35 \%$ & 4.694 & $0,37 \%$ & 3.555 & $0,35 \%$ \\
\hline 077-080 XIV. Enfermedades del sistema genitourinario & 9.567 & $2,47 \%$ & 521 & $0,80 \%$ & 8.615 & $0,69 \%$ & 6.080 & $0,59 \%$ \\
\hline $081 \mathrm{XV}$. Embarazo, parto y puerperio & 18 & $0,00 \%$ & 18 & $0,03 \%$ & 646 & $0,05 \%$ & 574 & $0,06 \%$ \\
\hline 082 XVI. Afecciones originadas en el periodo perinatal & 942 & $0,24 \%$ & 941 & $1,44 \%$ & 1.580 & $0,13 \%$ & 45.847 & $4,45 \%$ \\
\hline $\begin{array}{l}\text { 083-085 XVII. Malformaciones congénitas, deformidades } \\
\text { y anomalías cromosómicas }\end{array}$ & 962 & $0,25 \%$ & 870 & $1,33 \%$ & 13.622 & $1,09 \%$ & 35.138 & $3,41 \%$ \\
\hline $\begin{array}{l}\text { 086-089 XVIII. Síntomas, signos y hallazgos anormales } \\
\text { clínicos y de laboratorio, NCOP (1) }\end{array}$ & 10.969 & $2,83 \%$ & 1.896 & $2,90 \%$ & 40.449 & $3,23 \%$ & 37.457 & $3,64 \%$ \\
\hline 090-102 XX. Causas externas de mortalidad & 16.902 & $4,36 \%$ & 9.681 & $14,79 \%$ & 297.003 & $23,69 \%$ & 256.097 & $24,87 \%$ \\
\hline
\end{tabular}

(1) Por motivos de comparabilidad, el Registro de Defunciones incluye el VIH+ (R75) en el Capítulo I. Enfermedades infecciosas y parasitarias aunque la CIE-10 lo encuadra en el Capítulo XVIII (Síntomas, signos y hallazgos anormales clínicos y de laboratorio, NCOP).

Fuente: Elaboración propia a partir del Registro de Defunciones por Causa de Muerte (año 2005).

externas de muerte y tumores. El grupo que representa mayores pérdidas son las causas externas, con un coste estimado de 2.585 millones de euros (el 28,3\% del total). Los tumores representan unas pérdidas estimadas en 2.387 millones de euros (el 26,1\% del total). La razón de que las pérdidas ocasionadas por las causas externas superen a los generados por los tumores, pese a que los Años Potenciales de Vida Laboral Perdidos son superiores en estos últimos, tiene su explicación en la composición de los fallecimientos y en la dinámica del mercado laboral. Un $81,4 \%$ de los fallecimientos en edades previas a los 65 años por causas externas (un $81,9 \%$ de los APVLP) se producen en varones, mientras que este porcentaje baja al 64,6\% (al $60,1 \%$ ) en el caso de tumores. Sumando a esta circunstancia el hecho de que las tasas de empleo y los salarios en varones son mayores que las tasas de empleo y salarios para mujeres, cualquiera que sea el tramo de edad considerado, podemos explicar por qué las pérdidas ocasionadas por las causas externas de fallecimiento superan a las de los tumores. Las enfermedades del sistema circulatorio aparecen en tercer lugar representando un coste de 1.226 millones de euros (el 13,4\% del total). La edad media de fallecimiento por este grupo de enfermedad es notoriamente mayor que por tumores y por causas externas. De ahí, que los APVP y los APVLP sean notoriamente inferiores que los mismos conceptos de los dos grupos de enfermedades referidos y el impacto sobre la productividad, aun siendo muy elevado, tenga un menor peso. 
Tabla 4

Pérdidas de productividad laboral ocasionadas por muerte prematura Valores absolutos y distribución según causa del fallecimiento

\begin{tabular}{|c|c|c|c|c|c|c|}
\hline & \multicolumn{2}{|c|}{\begin{tabular}{|c|} 
Caso base \\
tasa anual de \\
descuento=3\%; \\
tasa anual de crecimiento de \\
la productividad $=1 \%$
\end{tabular}} & \multicolumn{2}{|c|}{$\begin{array}{c}\text { Tasa anual } \\
\text { de descuento }=0 \% ; \\
\text { tasa anual de crecimiento de } \\
\text { la productividad }=2 \%\end{array}$} & \multicolumn{2}{|c|}{$\begin{array}{c}\text { Tasa anual } \\
\text { de descuento }=6 \% \\
\text { tasa anual de crecimiento de } \\
\text { la productividad }=0 \%\end{array}$} \\
\hline & Valores $(€)$ & $\%$ & Valores $(€)$ & $\%$ & Valores $(€)$ & $\%$ \\
\hline Todas las causas & 9.136 .350 & $100,00 \%$ & 15.115 .025 & $100,00 \%$ & 6.649 .421 & $100,00 \%$ \\
\hline Enfermedades infecciosas y parasitarias & 528.848 & $5,79 \%$ & 819.525 & $5,42 \%$ & 391.251 & $5,88 \%$ \\
\hline Tumores & 2.386 .820 & $26,12 \%$ & 3.326 .692 & $22,01 \%$ & 1.902 .721 & $28,61 \%$ \\
\hline Enf. de sangre y de órg. Hematopoyéticos & 35.498 & $0,39 \%$ & 68.501 & $0,45 \%$ & 23.018 & $0,35 \%$ \\
\hline Enfermedades endocrinas, nutricionales y metabólicas & 126.188 & $1,38 \%$ & 213.592 & $1,41 \%$ & 90.765 & $1,37 \%$ \\
\hline Trastornos mentales y del comportamiento & 83.816 & $0,92 \%$ & 129.109 & $0,85 \%$ & 63.881 & $0,96 \%$ \\
\hline Enf. del sistema nervioso y de los órganos de los sentidos & 280.926 & $3,07 \%$ & 529.407 & $3,50 \%$ & 186.669 & $2,81 \%$ \\
\hline Enfermedades del sistema circulatorio & 1.226 .471 & $13,42 \%$ & 1.720 .991 & $11,39 \%$ & 975.782 & $14,67 \%$ \\
\hline Enfermedades del sistema respiratorio & 373.540 & $4,09 \%$ & 586.423 & $3,88 \%$ & 280.865 & $4,22 \%$ \\
\hline Enfermedades del sistema digestivo & 480.486 & $5,26 \%$ & 655.261 & $4,34 \%$ & 383.618 & $5,77 \%$ \\
\hline Enfermedades de la piel y del tejido subcutáneo & 5.025 & $0,05 \%$ & 6.786 & $0,04 \%$ & 4.146 & $0,06 \%$ \\
\hline Enf. del sistema osteomuscular y del tejido conjuntivo & 27.122 & $0,30 \%$ & 42.664 & $0,28 \%$ & 20.036 & $0,30 \%$ \\
\hline Enfermedades del sistema genitourinario & 47.344 & $0,52 \%$ & 68.957 & $0,46 \%$ & 36.850 & $0,55 \%$ \\
\hline Embarazo, parto y puerperio & 3.380 & $0,04 \%$ & 5.610 & $0,04 \%$ & 2.274 & $0,03 \%$ \\
\hline Afecciones originadas en el periodo perinatal & 335.989 & $3,68 \%$ & 883.161 & $5,84 \%$ & 156.648 & $2,36 \%$ \\
\hline Malformaciones congénitas & 259.451 & $2,84 \%$ & 639.968 & $4,23 \%$ & 132.017 & $1,99 \%$ \\
\hline Síntomas, signos, NCOP,... & 350.614 & $3,84 \%$ & 602.372 & $3,99 \%$ & 248.433 & $3,74 \%$ \\
\hline Causas externas & 2.584 .835 & $28,29 \%$ & 4.816 .005 & $31,86 \%$ & 1.750 .447 & $26,32 \%$ \\
\hline
\end{tabular}

Fuente: Elaboración propia a partir. Unidades: miles de euros.

Los tres siguientes grupos de mayor impacto sobre la productividad son las enfermedades infecciosas y parasitarias (529 millones de euros; un 5,79\% de las pérdidas de productividad laboral ocasionadas por los fallecimientos prematuros), junto con las enfermedades del sistema digestivo (480 millones de euros; un $5,26 \%$ ), y las enfermedades respiratorias (374 millones de euros; un 4,09\%).

En la Tabla 4 se representan también resultados obtenidos de aplicar una tasa anual de descuento del $6 \%$ en las pérdidas estimadas y considerar que la tasa de crecimiento de la productividad laboral sería nula y el resultado de aplicar una tasa de descuento nula sobre las pérdidas estimadas y una tasa anual de crecimiento de la productividad del $2 \%$. Las pérdidas estimadas oscilarían entre los 6.649 millones de euros y los 15.115 millones de euros.

En la Tabla 5 se muestran las pérdidas de producción estimadas por Comunidad Autónoma, junto a los APVP y los APVLP y a indicadores poblacionales, todo ello en términos relativos. De este modo se puede comparar el impacto relativo que presenta la pérdida de producción de cada región y se puede comparar dicho impacto con diferentes indicadores de peso relativo poblacional (población total, población activa y población ocupada). Para cada CCAA se ajustaron los datos de muertes prematuras por las tasas de empleo y los salarios de cada región, ajustando por edad y género. Así, de los 9.136 millones de euros estimados como pérdida para el caso base, un $95,7 \%$ se puede distribuir entre las diferentes CCAA, mien- 
Tabla 5

Distribución de los APVP, APVLP, pérdidas de productividad laboral ocasionadas por muerte prematura e indicadores poblacional por Comunidad Autónoma

\begin{tabular}{|l|r|r|r|r|r|c|}
\hline & APVP & APVLP & $\begin{array}{c}\text { Pérdida laboral } \\
\text { (caso base) }\end{array}$ & $\begin{array}{c}\text { Población } \\
\text { total }\end{array}$ & $\begin{array}{c}\text { Población } \\
\text { activa }\end{array}$ & $\begin{array}{c}\text { Población } \\
\text { ocupada }\end{array}$ \\
\hline Andalucía & $18,58 \%$ & $18,95 \%$ & $15,45 \%$ & $17,82 \%$ & $16,45 \%$ & $15,60 \%$ \\
\hline Aragón & $2,74 \%$ & $2,77 \%$ & $2,87 \%$ & $2,88 \%$ & $2,89 \%$ & $3,00 \%$ \\
\hline Asturias (Principado de) & $2,73 \%$ & $2,65 \%$ & $2,37 \%$ & $2,44 \%$ & $2,16 \%$ & $2,14 \%$ \\
\hline Balears (Illes) & $2,15 \%$ & $2,18 \%$ & $2,07 \%$ & $2,24 \%$ & $2,42 \%$ & $2,47 \%$ \\
\hline Canarias & $4,93 \%$ & $5,00 \%$ & $3,96 \%$ & $4,45 \%$ & $4,53 \%$ & $4,41 \%$ \\
\hline Cantabria & $1,23 \%$ & $1,20 \%$ & $1,07 \%$ & $1,28 \%$ & $1,25 \%$ & $1,26 \%$ \\
\hline Castilla y León & $5,53 \%$ & $5,40 \%$ & $4,99 \%$ & $5,70 \%$ & $5,36 \%$ & $5,39 \%$ \\
\hline Castilla-La Mancha & $3,94 \%$ & $3,94 \%$ & $3,39 \%$ & $4,32 \%$ & $4,03 \%$ & $4,03 \%$ \\
\hline Cataluña & $15,42 \%$ & $15,16 \%$ & $18,36 \%$ & $15,81 \%$ & $16,94 \%$ & $17,35 \%$ \\
\hline Comunitat Valenciana & $11,42 \%$ & $11,32 \%$ & $10,15 \%$ & $10,55 \%$ & $10,78 \%$ & $10,82 \%$ \\
\hline Extremadura & $2,37 \%$ & $2,35 \%$ & $1,61 \%$ & $2,47 \%$ & $2,19 \%$ & $2,03 \%$ \\
\hline Galicia & $7,10 \%$ & $6,81 \%$ & $6,14 \%$ & $6,26 \%$ & $6,01 \%$ & $5,96 \%$ \\
\hline Madrid (Comunidad de) & $11,87 \%$ & $12,12 \%$ & $16,81 \%$ & $13,55 \%$ & $14,69 \%$ & $15,07 \%$ \\
\hline Murcia (Región de) & $2,94 \%$ & $3,19 \%$ & $2,81 \%$ & $3,04 \%$ & $2,97 \%$ & $3,00 \%$ \\
\hline Navarra (Comunidad Foral de) & $1,18 \%$ & $1,17 \%$ & $1,36 \%$ & $1,35 \%$ & $1,39 \%$ & $1,45 \%$ \\
\hline País Vasco & $4,89 \%$ & $4,70 \%$ & $5,59 \%$ & $4,86 \%$ & $4,95 \%$ & $5,05 \%$ \\
\hline Rioja (La) & $0,65 \%$ & $0,71 \%$ & $0,67 \%$ & $0,69 \%$ & $0,72 \%$ & $0,74 \%$ \\
\hline Ceuta y Melilla & $0,33 \%$ & $0,38 \%$ & $0,31 \%$ & $0,32 \%$ & $0,28 \%$ & $0,25 \%$ \\
\hline Total CCAA & $100,00 \%$ & $100,00 \%$ & $100,00 \%$ & $100,00 \%$ & $100,00 \%$ & $100,00 \%$ \\
\hline
\end{tabular}

Fuente: Elaboración propia.

tras que queda un 4,3\% de difícil asignación directa. Por tanto, los porcentajes que muestra la Tabla 5, se refieren a los 8.740 millones de euros (el citado $95,7 \%$ sobre el total de 9.136 millones de euros) que sí son claramente imputables a cada una de las diferentes regiones.

\section{DISCUSIÓN}

En este trabajo, se ha estimado que las pérdidas de producción para la economía española que causan las muertes prematuras ascienden a 9.136 millones de euros, con un arco que oscila entre los 6.649 y los 15.115 millones de euros, dependiendo de las tasas de descuento y de crecimiento de la productividad consideradas. Ante la magnitud de la cifra estimada, resulta conveniente compararla con otros indicadores para revelar su verdadero coste de oportunidad. Así, esta cifra sería equivalente a un $1,01 \%$ del Producto Interior Bruto de Espa- ña en el año tomado como referencia, o a un $18,25 \%$ del gasto sanitario público del mismo periodo.

Como puede observarse en la tabla 3, si se seleccionan los fallecimientos a cualquier edad, las enfermedades del sistema circulatorio y los tumores son los grupos de mayor peso. Ello sigue siendo cierto si se selecciona los fallecimientos en edades menores de los 65 años, si bien la importancia relativa cambia (ahora el mayor peso de la distribución se concentra en los tumores seguidos de las enfermedades del sistema circulatorio), apareciendo un tercer grupo (causas externas). Cuando el foco de atención se desplaza a los Años Potenciales de Vida Perdidos (APVP), los tumores ocupan el primer lugar seguidos ahora en importancia por las causas externas y quedando en tercer lugar las enfermedades del sistema circulatorio. Finalmente, cuando analizamos los Años Potenciales de Vida Laboral Perdidos (APVLP), los tumores siguen ocu- 
pando el primer lugar, si bien la distancia con las causas externas de mortalidad se ha reducido y ha aumentado respecto a las enfermedades del sistema circulatorio (tabla 2).

Si acudimos a la lista reducida de enfermedades (Clasificación Internacional de Enfermedades- $10^{\text {a }}$ revisión), la causa de muerte que mayor impacto ocasiona en España son los accidentes de tráfico con 120.091 APVP (el 9,6\% el total), 105.242 APVLP (el 10,2\% del total) y 1.046,7 millones de euros en pérdidas (el 11,5\% del total). En importancia le seguiría el tumor maligno de la tráquea, de los bronquios y del pulmón, con cifras de impacto relativo del 7,4\% de los APVP, 6,0\% de los APVLP y $6,0 \%$ de las pérdidas laborales estimadas. En tercer lugar se encontraría los fallecimiento por suicidios y lesiones autoinfligidas, con cifras del 4,9\% de los APVP, 5,1\% de los APVLP y 5,8\% de las pérdidas laborales estimadas. Asimismo, se debe señalar la importante carga del tumor maligno de la mama en mujeres (responsable de un 10,5\% de los APVP, un 9,2\% de los APVLP y un $8,9 \%$ de las pérdidas laborales femeninas) y del infarto agudo de miocardio en el caso de los hombre $(5,6 \%$ de los APVP, 4,8\% de los APVP y $4,7 \%$ de las pérdidas laborales masculinas).

La pérdida de productividad entre varones y mujeres se distribuye de manera desigual: mientras que los varones soportan un $83,9 \%$ de la misma, sobre las mujeres recae un $16,1 \%$. Ello se debe tanto a factores epidemiológicos como a factores sociolaborales. Por una parte, los fallecimientos prematuros de varones son muy superiores a los de las mujeres. Así, un 70,8\% de los fallecimientos en edad laboral o previa (menores de 65 años) y un 70,0\% de los Años Potenciales de Vida Laboral Perdidos son soportados por varones. Por otra parte, las tasas de participación laboral y los salarios femeninos son inferiores a los masculinos para cualquier tramo de edad considerado. Por tanto, al fallecimiento de un varón de, por ejemplo, 50 años, le corresponde una mayor pérdida de productividad laboral que la correspondiente a una mujer de la misma edad.

La incorporación de la producción no laboral perdida (producción doméstica) implicaría, por una parte, un incremento en la partida del coste total estimado y, por otra, una mayor reparto del coste entre varones y mujeres, si bien hemos de subrayar que las diferencias epidemiológicas son tan fuertes que los varones seguirían soportando una mayor carga.

Asimismo, cuando se realiza la distribución de las pérdidas laborales por CCAA, se puede observar que varias CCAA presentan unas pérdidas relativas medidas en APVP y APVLP elevadas en relación con su peso poblacional (Andalucía, Galicia, Canarias y Comunidad Valenciana), mientras en la Comunidad de Madrid ocurre justo lo contrario. Ello es consecuencia directa de factores epidemiológicos que se asocian con un mayor o menor peso de los fallecimientos prematuros en estas regiones. Sin embargo, si observamos directamente las pérdidas laborales, vemos que Cataluña y Madrid son las dos Comunidades con mayores pérdidas. Ello es coherente con el hecho de que en estas dos regiones tanto los salarios como las tasas de empleo son notoriamente mayores que la media nacional y, por tanto, ante una muerte prematura mayor es la probabilidad de que la persona fallecida estuviera trabajando $\mathrm{y}$ mayor sería la pérdida de productividad (medida por el salario) estimada. Ello mismo ocurre, aunque en menor grado en el caso del País Vasco. En aquellas regiones con menores tasas de empleo y menores salarios respecto a la media, ocurre el caso contrario. Así, por ejemplo, en Andalucía, pese a sus peores resultados relativos en términos de APVP y APVLP respecto a su población total, el peso de su pérdida laboral respecto al total de las CCAA queda 
suavizado. Por tanto, podemos concluir en la complementariedad de los tres indicadores manejados a la hora de aportar información relevante sobre el impacto laboral de los fallecimientos prematuros.

Existen otros trabajos de referencia donde se estima el coste global de la enfermedad, distinguiendo tanto la parte de costes directos sanitarios como las pérdidas de producción laboral ocasionadas por la enfermedad ${ }^{11,12}$. En el trabajo estadounidense $\mathrm{e}^{11}$, un $60,7 \%$ de los costes estimados corresponden a costes sanitarios y el $39,3 \%$ restante a pérdidas laborales $(39,3 \%)$. De estos últimos, el $72 \%$ de los costes son debidos a fallecimientos prematuros. Las enfermedades cardiovasculares representaron la principal partida de pérdidas laborales ocasionadas por mortalidad prematura con un peso relativo del $20,9 \%$, seguido de cerca de los tumores $(20 \%)$. A un cajón de sastre denominado "Otras enfermedades y fallecimientos sin asignar", correspondería el 33,9\% de las pérdidas laborales ocasionadas por mortalidad prematura. Aunque no se especifica con claridad, en esta partida figura (por exclusión) las causas externas de mortalidad. A bastante distancia de los grupos de enfermedades citados se encontrarían las enfermedades respiratorias (un 6,4\%), las enfermedades infecciosas y parasitarias $(5,1 \%)$ y las enfermedades del aparato digestivo $(4,5 \%)$. Por lo que respecta al trabajo canadiense ${ }^{12}$, el $52,7 \%$ del coste total estimado total se corresponde con costes directos sanitarios y el $47,3 \%$ restante son imputables a costes no sanitarios. La pérdida de producción imputable a mortalidad prematura correspondía a un $21 \%$ del coste total (un $44 \%$ de los costes no sanitarios). Distinguiendo por categorías, la partida de mayor peso corresponde a cáncer con un $31,7 \%$, seguida de enfermedades cardiovasculares, con un 24,6\%, y, en tercer lugar de causas externas, con un $17,7 \%$. A distancia de los grupos de enfermedades citados se encontrarían las enfer- medades respiratorias (un 4,9\%) y las enfermedades del aparato digestivo $(3,4 \%)$.

Por tanto, por encima de diferencias metodológicas existentes entre el presente trabajo y los estudios canadiense y estadounidense, parece existir patrones comunes en los tres casos: en primer lugar, la gran importancia de los tumores y las enfermedades del sistema circulatorio como principales causas de muerte prematura; en segundo lugar, los tres grupos de enfermedades de mayor impacto en relación a la pérdida de productividad son los tumores, las causas externas y las enfermedades del sistema circulatorio; en tercer lugar, la explicación de que las causas externas causen tan elevadas pérdidas en los tres estudios reside en el hecho de que los fallecidos son personas de edades mucho más jóvenes que en los dos grupos referidos, por lo que el flujo de producción perdida por fallecimiento es mucho mayor. En cuanto a las diferencias, la ordenación de los grupos de mayor impacto sobre las pérdidas laborales cambia entre países. No obstante, esta no es una circunstancia extraña, dadas las diferencias epidemiológicas entre Canadá, EEUU y España ${ }^{13}$.

En relación con el impacto de las pérdidas de producción sobre el total de la economía, sí existe coincidencia entre los informes estadounidense y canadiense. A partir de la información referida en los informes, se puede estimar que las pérdidas laborales ocasionadas por enfermedades y accidentes ascendieron al 4,85\% del Producto Interior Bruto (PIB) de EEUU en el año 2004 y un 4,4\% del PIB canadiense en el año 1998. En un trabajo realizado recientemente y centrado en Cataluña ${ }^{14}$, la suma de las pérdidas de producción laboral ocasionadas por los fallecimientos prematuros y por las situaciones de Incapacidad (Permanente y Temporal) supusieron el equivalente al 4,7\% del PIB de Cataluña en el periodo de referencia (año 2004), cifra lige- 
ramente superior al presupuesto sanitario público de Cataluña en el mismo año ${ }^{15}$.

A las anteriores cifras, podríamos añadir los costes del sistema de cuidados sociales (o formales) a personas que están en situación de discapacidad a consecuencia de una enfermedad y los costes soportados por la red familiar de apoyo informal. Estos costes pueden ser especialmente elevados en enfermedades discapacitantes como son las enfermedades neurodegenerativas (demencias, enfermedad de Parkinson, esclerosis múltiple, accidentes cerebrovasculares), las enfermedades osteomusculares y las enfermedades cardiovasculares (cardiopatías isquémicas e insuficiencia cardíaca crónica). Existe una abundante literatura sobre cuidados informales, si bien hasta el momento los estudios se han centrado en grupos concretos de pacientes que soportan una determinada enfermedad y apenas existen trabajos que estimen el coste total que suponen para la sociedad el conjunto de cuidados informales prestados. No obstante, un estudio recientemente publicado cifra en España el coste social de los cuidados informales en cifras cercanas al 1,5\% del PIB en el año $2002^{16}$.

Como señalábamos en la introducción, los recursos no deberían asignarse en función del impacto de una determinada enfermedad, sino allí donde una intervención produzca mayores beneficios en términos de salud. A pesar de las limitaciones que suponen los estudios de coste de la enfermedad, los gobiernos de muchos países y regiones siguen promoviendo su realización. La razón de ello es que los decisores públicos consideran que la información sobre el impacto económico que generan las enfermedades y problemas de salud puede ser una herramienta útil de cara a la planificación de sus programas ${ }^{17}$. Dicha información no sustituye sino complementa a la información epidemiológica sobre problemas de salud de las poblaciones. Como señala Knapp ${ }^{18}$, "Los, a menudos sustancia- les, costes no sanitarios no son elementos decorativos de obscuros estudios académicos... Son una carga real sobre recursos que deben ser soportados por algunos individuos o por parte de la sociedad". Sin embargo, "las estimaciones de los costes de la enfermedad meramente describen lo que es, no lo que debe hacerse (por arte de los decisores sanitarios)... con sus limitados recursos". Por tanto, la utilidad de un estudio de costes consiste en señalar el impacto económico de una determinada enfermedad, revelando aquellos costes que no eran visibles o sobre los que se desconocían su cuantía en un principio.

Varias autoridades sanitarias españolas han mostrado recientemente interés en incorporar estudios de coste de la enfermedad como soporte a su toma de decisiones $^{19,20}$. Asimismo, el Ministerio de Sanidad y Consumo ha incluido estimaciones sobre el coste de la enfermedad en los recientes Planes Integrales de abordaje, prevención y manejo clínico de la Diabetes Mellitus, las enfermedades isquémicas del corazón y los tumores ${ }^{21-23}$. Dicho interés sugiere que, una vez tomadas en cuenta las limitaciones de este tipo de estudios para la priorización de programas, y subrayando sus fortalezas a la hora de acercarnos al impacto social de las enfermedades, pueden ser una herramienta útil en la planificación de políticas públicas.

Así, esta información podría integrase dentro de la elaboración de un Plan de Salud, donde el primer paso consistiría en disponer de datos epidemiológicos suficientes para identificar los problemas de salud claves de la región o país considerado. En segundo lugar, se afrontaría el cálculo del coste que ocasionan estas enfermedades y problemas a la población (empleando un concepto amplio de coste), como una aproximación a la pérdida de bienestar social que ocasionan las mismas. Un tercer paso sería el disponer de información sobre los medios técnicos y humanos que pueden 
aplicarse en políticas o intervenciones que incidan sobre estos problemas. Tras estos pasos, la secuencia lógica sería la identificación de aquellos programas e intervenciones transversales eficientes, es decir, aquellas políticas que permitan mejorar la esperanza y la calidad de vida de la población a partir del menor sacrificio de recursos disponibles. El siguiente paso sería la puesta en marcha de dichas intervenciones y su posterior evaluación.

Así pues, una selección de las actuaciones transversales sobre la salud (sanitarias, laborales, educativas, viales, etc.) que demuestren científicamente su efectividad y eficiencia para mejorar la situación de la población condicionada por los problemas de salud estudiados puede constituir una herramienta de gran valor para dar soporte a la toma de decisiones sobre asignación de recursos en políticas de salud (Políticas Basadas en la "Evidencia"). Lógicamente, la puesta en funcionamiento o la potenciación de estos programas pueden derivar en un mayor coste sanitario, si bien habría que estudiar qué ganamos a cambio (por ejemplo, en términos de pérdidas económicas evitadas o en términos de años de vida salvados). En último término, elegir es inevitable y la consideración de elementos de racionalidad económica en el proceso puede ayudar a que las elecciones sean más racionales y la asignación de los recursos más transparente y eficiente.

Asimismo, quiero agradecer los comentarios recibidos por un evaluador anónimo, los cuales han contribuido a enriquecer el trabajo.

\section{BIBLIOGRAFÍA}

1. Grossman, M. The Demand for Health: A Theoretical and Empirical Investigation. New York: Columbia University Press; 1972.

2. Grossman M The Human Capital Model of the Demand for Health. In AJ. Culyer and JP Newhou- se (eds.). Handbook of Health Economics, Amsterdam: North-Holland-Springer-Verlag; 2000.

3. Suhrcke M, McKee M, Sauto Arce R, Tsolova S, Mortensen J. The contribution of health to the economy in the European Union. European Communities. Luxembourg: Office for Official Publications of the European Communities; 2005

4. Comisión Europea. Accesible en http://ec.europa.eu/health/ph_information/indicators/lifeyears_en.htm. Última fecha de acceso 4 de junio de 2008.

5. Commission of the European Communities. "White Paper-Together for Health: A Strategic Approach for the EU 2008-2013”. Brussels, 2007. Accesible en: http://ec.europa.eu/health/ph_overview/Documents/strategy_wp_en.pdf. Última fecha de acceso 11 de julio de 2008 .

6. Becker GS. Human Capital. Chicago: Columbia University Press; 1964.

7. Mincer J. Schooling, Experience, and Earnings. Human Behavior \& Social Institutions. New York: Columbia University Press; 1974

8. Hodgson TA, Meiners MR. (1982). "Cost-of-illness methodology: a guide to assessment practices and procedures". Milbank Mem Fund Q. 1982; 60: 429-91.

9. Max W, Rice DP, Mackenzie EJ. The lifetime cost of injury. Inquiry 1990; 27: 332-343.

10. Robinson JC."Philosophical Origins of the Economic Valuation of Life". Milbank Q; 1986, 64:133-55.

11. National Institutes of Health. National Heart, Lung and Blood Institute. Fact Book. Fiscal Year 2003.

12. Minister of Public Works and Government Services Canada. Economic Burden of Illness in Canada, 1998. Ottawa, Ontario, 2002.

13. Organización Mundial de la Salud/World Health Organization. Burden of Disease statistics Disponible en http://www.who.int/healthinfo/bod/en/index. html (acceso el día 19 de octubre de 2008).

14. Oliva J. Pérdidas de producción laboral ocasionadas por las enfermedades y problemas de salud en Cataluña. Presupuesto y Gasto Público, 2008; 53 (4): 119-147.

15. Ministerio de Sanidad y Consumo. Estadística de Gasto Sanitario Público. Accesible en www.msc.es (consultado el 15 de abril de 2008). 
16. Oliva J, Osuna R, Jorgensen N. Una estimación de los costes de los cuidados informales en España, en Sergi Jiménez (coordinador) Aspectos económicos de la dependencia y el cuidado informal en España. Madrid, 2008.

17. Hu TW (2006). Perspectives: an international review of the national cost estimates of mental illness, 1990-2003. J Ment Health Policy Econ, 2006; 9(1): 3-13.

18. Knapp M (2003). Hidden costs of mental illness. Br. J. Psychiatry; 183: 477-478.

19. Plan de Salud de Canarias 2004-2008. Servicio Canario de la Salud. Consejería de Sanidad del Gobierno de Canarias.
20. Gisbert R, Brosa M, Bohigas Ll. Distribución del presupuesto sanitario público de Cataluña del año 2005 entre las 17 categorías CIE-9-MC. Gaceta Sanitaria 2007, 21 (2): 124-131.

21. Estrategia en Diabetes del Sistema Nacional de Salud. Ministerio de Sanidad y Consumo, 2007.

22. Estrategia en Cáncer del Sistema Nacional de Salud. Ministerio de Sanidad y Consumo, 2006.

23. Estrategia en Cardiopatía Isquémica del Sistema Nacional de Salud. Ministerio de Sanidad y Consumo, 2006. 
